\title{
Associação entre depressão, níveis de dor e falta de apoio social em pacientes internados em enfermarias de clínica médica
}

\author{
Association of depression, levels of pain and lack of social \\ support in patients admitted to general medical wards \\ Camila Andrade Marques', Betina Stefanello', Camila Nemoto de Mendonça', Letícia Maria Furlanetto ${ }^{1}$
}

\section{RESUMO}

Objetivo: Verificar a associação entre depressão, níveis de dor e falta de apoio social em pacientes clínicos internados. Métodos: Em um estudo transversal, 1.147 adultos admitidos nas enfermarias de clínica médica de um hospital universitário foram selecionados por randomização e avaliados durante a primeira semana de internação. Foram utilizados: Subescala Cognitivo-afetiva do Inventário Beck de Depressão (BDI-13), Índice Charlson de Comorbidade Física e escalas numéricas para avaliar dor e percepção de gravidade física. Foram considerados deprimidos os pacientes que pontuaram acima de 10 no BDI-13. Investigou-se apoio social por meio da pergunta direta: "Com quantos parentes ou amigos você se sente à vontade e pode falar sobre tudo ou quase tudo?". Foram considerados como tendo falta de apoio social os pacientes que relataram ter menos que quatro parentes ou amigos confidentes. Foram utilizados os testes T de Student, Qui-quadrado e Regressão Logística. Resultados: Dos 1.147 pacientes, 25,3\% apresentavam depressão. Escolaridade [odds ratio (OR): 0,96; intervalo de confiança (IC): 0,89-0,96; $p<0,001$, renda familiar (OR: 0,92; IC: 0,86-0,99; $p=0,018$ ), maior intensidade de dor (OR: 1,04; IC: 1,00-1,08; $p=0,036$ ), falta de apoio social (OR: 2,02; IC:

\section{Palavras-chave}

Depressão, dor, apoio social, pacientes internados, hospital geral.
1,49-2,72; $\mathrm{p}<0,001$ ) e percepção de maior gravidade física (OR: 1,07; IC: 1,02-1,13; $\mathrm{p}=0,008$ ) se associaram independentemente à depressão. Conclusão: Pacientes clínicos deprimidos relatam mais falta de apoio social e dor, mesmo após controlar para variáveis confundidoras sociodemográficas e clínicas.

\begin{abstract}
Objective: To evaluate the association of depression, levels of pain and lack of social support in medical inpatients. Methods: In a cross sectional observational study, 1,147 adults admitted to the general medical wards of a university hospital were randomized and evaluated during the first week of admission. The following instruments were used: cognitive-affective subscale of the Beck Depression Inventory (BDI-13), Charlson Comorbidity Index and numerical scales to evaluate pain and perception of medical burden. Patients who scored $>10$ in the BDI-13 were considered depressed. Social support was investigated asking the
\end{abstract}

1 Universidade Federal de Santa Catarina (UFSC), Centro de Ciências da Saúde, Departamento de Clínica Médica, Florianópolis, SC, Brasil.

Endereço para correspondência: Letícia Maria Furlanetto

Av. Rio Branco, 333/804, Centro - 88015-200 - Florianópolis, SC, Brasil

Telefax: (+55 48) 3234-9756

E-mail: leticiafurlanetto@yahoo.com.br 


\section{Keywords}

Depression, pain, social support, medical inpatients, general hospital. following question: "How many relatives or friends do you feel at easy and can talk about almost everything?". Those who had less than four relatives or close friends were considered as having lack of social support. The Student T test, Chi-square test and Logistic Regression analysis were used. Results: Of the 1,147 patients that comprised the sample, 25.3\% had depression. Educational level [odds ratio (OR): 0.96; confidence interval (CI): 0.89-0.96; $\mathrm{p}<0.001$ ], household income (OR: 0.92; Cl: 0.86-0.99; $p=0.018$ ), pain levels (OR: 1.04; Cl: 1.00-1.08; $p=$ 0.036), lack of social support (OR: 2.02; Cl: 1.49-2.72; $\mathrm{p}<0.001)$ and perception worse physical illness severity (OR: 1.07; Cl: 1.02-1.13; $p=0.008$ ) were independently associated with depression. Conclusion: Depressive medical inpatients report more lack of social support and pain even after controlling for social, demographic and clinical variables.

\section{INTRODUÇÃO}

Pacientes com transtornos do humor relatam maiores níveis de dor'. A prevalência de episódios depressivos entre pacientes clínicos com dor é alta (42\%)². Pacientes com comorbidade de doenças físicas e depressão apresentam mais dor crônica e têm pior evolução apesar do tratamento adequado3. Observou-se que pacientes com artrite, portanto com dor crônica, têm maior predisposição de desenvolver transtornos do humor'. Estudos recentes vêm demonstrando mecanismos inflamatórios que exercem papel na fisiopatologia tanto da depressão como da dor e de diversas outras doenças sistêmicas ${ }^{5}$. Em pacientes com artrite reumatoide, a depressão e a inflamação (medida por meio da dosagem da proteína ( reativa) têm correlação entre si, mas influenciam a dor de forma independente 6 . De maneira semelhante, em pacientes com câncer de mama, o tratamento da depressão foi efetivo para reduzir os indicadores de inflamação. Contudo, ainda existem poucos estudos no Brasil sobre a interface da depressão com a dor e outros aspectos psicossociais.

Uma pesquisa realizada na comunidade revelou que os indivíduos que relatavam ter dor apresentavam mais transtornos depressivos e ansiosos, além de terem pior qualidade de vida, maior incapacidade, maior utilização dos cuidados de saúde e uso de substâncias ${ }^{8}$.

Por ser um achado subjetivo do exame clínico, a percepção da dor depende não só das vias biológicas (como o que ocorre na inflamação), mas também das características psicológicas do paciente. Um ensaio clínico mostrou que pacientes com dor crônica e com padrões negativos de pensamento (como o catastrófico) perceberam maiores níveis de dor em relação àqueles que tinham como padrão de pensamento a aceitação da dor ${ }^{9}$. Outra pesquisa evidenciou a associação entre a gravidade da depressão e a intensidade do pensamento catastrófico em pacientes com anemia falciforme ${ }^{10}$. Entre pacientes com dor coronariana aguda a depressão persistente vem sendo associada com aumento na mortalidade e na morbidade cardíaca"1. Ou seja, apesar de repetidos estudos sobre o assunto, os mecanismos psicológicos e fisiológicos da dor e sua associação com depressão não estão esclarecidos e ainda são objeto de controvérsia.
Discute-se, também, o papel do apoio social no aparecimento, manutenção ou melhora da depressão. Ambos - apoio social e depressão - podem influenciar, direta ou indiretamente, na evolução e bem-estar de pacientes clínicos, como observado entre pacientes com infarto agudo do miocárdio (IAM) ${ }^{12}$. A depressão mostrou-se um fator preditor de mortalidade após um ano de IAM, porém o apoio social não se relacionou diretamente com a sobrevivência. Contudo, altos níveis de apoio social se relacionaram com melhora nos sintomas depressivos, podendo ser, assim, um fator protetor indireto para pessoas com IAM ${ }^{12}$. Além disso, pacientes com insuficiência cardíaca deprimidos apresentaram mais pessimismo, baixa percepção de apoio social e um padrão de comportamento de evitação ${ }^{13}$. Ou seja, além de se isolarem, ainda não perceberam o apoio que tinham. Provavelmente esse mesmo fenômeno aconteceu com um grupo de pacientes em uso de terapia antirretroviral que, em um ano de seguimento, apresentou concomitantemente aumento na sintomatologia depressiva e diminuição nos níveis de apoio social ${ }^{14}$. Entre idosos com quadros dolorosos, a rede social e o estado funcional parecem estar mais relacionados com a depressão do que com a dor objetiva propriamente dita ${ }^{15}$. A associação entre apoio social e depressão também foi evidenciada em pacientes submetidos ao transplante de medula óssea ${ }^{16}$. Nesses pacientes, após seguimento de um ano, observou-se que o aumento no suporte social percebido esteve relacionado à redução nos níveis de depressão ${ }^{16}$.

A depressão, a dor e a falta de apoio social são realidades recorrentes na prática clínica e interferem diretamente no diagnóstico e tratamento de diversas patologias. Vale salientar a importância do tratamento e do acompanhamento desses pacientes no contexto intra-hospitalar e após alta, pois já se evidenciou que os transtornos psiquiátricos em pacientes clínicos hospitalizados não remitem espontaneamente ${ }^{17}$. Ter história familiar psiquiátrica positiva e menor escolaridade já se mostrou relacionado com a persistência dos transtornos ansiosos, da depressão e dos transtornos somatoformes após a alta ${ }^{17}$. Identificar precocemente a combinação de diferentes riscos e instituir o tratamento adequado previne novos episódios de depressão ${ }^{18}$. 
Apesar da vasta literatura sobre o assunto, ainda não é possível definir exatamente o quanto essas variáveis interagem gerando sofrimento psíquico ou bem-estar, nem como isso acontece, nos pacientes clínicos. O objetivo deste estudo é verificar se existe associação entre depressão, níveis de dor e falta de apoio social em pacientes internados em enfermarias de clínica médica de um hospital universitário.

\section{MÉTODOS}

\section{Desenho e local}

Trata-se de um estudo transversal, desenvolvido nas Enfermarias de Clínica Médica do Hospital Universitário Professor Polydoro Ernani de São Thiago de Florianópolis, da Universidade Federal de Santa Catarina (HU-UFSC). A cidade de Florianópolis, localizada no estado de Santa Catarina, tem aproximadamente 450 mil habitantes, com 97\% localizados na área urbana. O Hospital Universitário é referência, atendendo à Grande Florianópolis e aos casos provenientes do interior do estado.

\section{Amostra}

Foram anotados os nomes de todos os pacientes consecutivamente internados nas Enfermarias de Clínica Médica do HU-UFSC desde 15 de abril de 2006 até 30 de junho de 2011, excluindo os meses não letivos (janeiro, fevereiro e julho), que no total somaram 7.093 pacientes. Embora o cálculo da amostra necessária para avaliar a prevalência de sintomatologia depressiva moderada a grave em pacientes clínicos, com estimativa de $30 \%$ e nível de significância de 0,05, tenha sido de 323 pacientes, com a finalidade de atingir os objetivos do estudo (associação entre variáveis), foi necessária uma amostra maior (superior a 1.000 indivíduos) até que os resultados ficassem constantes por causa das múltiplas análises realizadas. Por meio de uma lista de números randômicos, foram selecionados 1.630 pacientes, que foram abordados, esclarecidos sobre a natureza do estudo e convidados a participar. Esse número foi escolhido considerando que 29,6\% ( $N=483$ ) foram excluídos, sendo 259 por incapacidade física que impossibilitasse a realização de forma adequada da entrevista (por afasia, dispneia, prejuízo cognitivo etc.), 117 por causas administrativas (por exemplo, estar fora do leito realizando exames complementares, cirurgias etc.), 13 por estarem sendo reinternados e 94 por recusarem. Portanto, compuseram a amostra 1.147 pacientes que consentiram e que não preenchiam critérios de exclusão.

\section{Procedimentos}

Os pacientes foram avaliados antes de completar uma semana de internação. Os dados foram colhidos por alunos da graduação em Medicina já com formação em psicopatologia e sob supervisão direta da professora (LMF), com experiência em interconsulta há mais de 20 anos. Foram coletados dados sociodemográficos como idade, sexo, estado civil, escolaridade em anos, renda familiar em salários-mínimos e dados da história clínica, bem como preenchidos os questionários descritos a seguir, por meio de entrevista e dados do prontuário.

O Miniexame do Estado Mental (MMSE) foi utilizado com a finalidade de rastrear pacientes com prejuízo cognitivo, principalmente delirium e/ou demência ${ }^{19}$. É composto de 11 perguntas e requer somente de 5 a 10 minutos para ser administrado. O ponto de corte sugerido pelos autores da escala é de 23/24 para a suspeita de prejuízo cognitivo ${ }^{19}$. Contudo, pelo fato de pacientes com baixa escolaridade poderem ter pontuação inferior nessa escala, sem que apresentem prejuízo cognitivo ${ }^{20}$, quando apropriado, foram utilizados os pontos de corte de acordo com o proposto por Bertolucci et al. (13 para analfabetos; 18 para aqueles com 1 a 8 anos incompletos e 26 para pacientes com 8 ou mais anos de escolaridade $)^{21}$.

O Inventário Beck de Depressão (BDI) foi elaborado para medir a gravidade de depressão em adolescentes e adultos $^{22}$. Entretanto, vem sendo usado como instrumento de rastreamento de depressão. A subescala cognitivo-afetiva (primeiros 13 itens - BDI-13) mostrou-se válida em pacientes atendidos por causa de doenças físicas no nosso hospital ${ }^{23}$. Foram considerados como tendo depressão aqueles pacientes que pontuaram acima de 10 no BDI-13.

As percepções de gravidade física e de dor foram avaliadas usando-se escalas do tipo NRS (Numerical Rating Scales) ${ }^{24}$ de 11 pontos (0 a 10), sendo perguntado, respectivamente: "De zero a dez, qual número melhor descreve a gravidade de sua doença no momento? Zero (0) seria sem doença e dez (10) seria doença mais grave possível". E, "De zero a dez, qual número melhor descreve sua dor agora? Zero (0) seria 'sem dor' e dez (10) seria a dor mais forte possível".

Foi avaliado apoio social com a seguinte pergunta: "Com quantos parentes ou amigos você se sente à vontade e pode falar sobre quase tudo?" Essa pergunta foi validada em uma amostra brasileira por Chor et al..$^{25}$. Foram considerados como tendo falta de apoio social aqueles pacientes que relataram ter menos que quatro parentes ou amigos confidentes. Esse ponto de corte foi escolhido porque um estudo anterior mostrou que indivíduos com menos de quatro confidentes têm maior chance de evoluir com depressão ${ }^{26}$.

A gravidade física objetiva foi medida pelo Índice Charlson de Comorbidade Física. Esse instrumento foi elaborado para ser utilizado em estudos de avaliação de prognóstico, uma vez que é capaz de prever mortalidade. No cômputo desse item são avaliados não só o número de doenças, mas também a gravidade delas ${ }^{27}$.

\section{Análise estatística}

A análise estatística foi feita utilizando o programa SPSS (Statistical Package for Social Sciences), versão 17.0 para Windows. Foram considerados estatisticamente significativos os valores de $p \leq 0,05$. Quando foram feitas múltiplas comparações, 
foi utilizada a correção de Bonferroni. Para a análise descritiva dos dados, foram empregadas frequências, porcentagens, médias, medianas e desvios-padrão. Foi utilizado o teste T de Student para amostras independentes e o teste do Qui-quadrado, conforme apropriado, com o objetivo de comparar os pacientes deprimidos (BDI-13 > 10) e não deprimidos. Aqueles fatores que foram significativamente diferentes ( $p \leq$ $0,05)$ na análise univariada foram analisados na multivariada, por meio da Regressão Logística (com o modo "stepwise likelihood-ratio") conjuntamente com a gravidade física objetiva, para verificar sua associação independente com a depressão.

\section{Aspectos éticos}

O estudo foi aprovado pelo Comitê de Ética para Pesquisas com Seres Humanos da UFSC. Os pacientes foram orientados sobre o estudo e informados que em nada seria alterado seu tratamento caso a decisão fosse a de não participar. Aqueles que concordaram em participar assinaram o termo de consentimento livre e esclarecido. Caso fosse detectada qualquer alteração mental sugestiva de ter benefício de alguma intervenção, os pacientes e médicos-assistentes foram informados para que a interconsulta psiquiátrica pudesse ser solicitada, se julgassem necessário.

\section{RESULTADOS}

Como já dito, a amostra foi composta por 1.147 pacientes. Desses, a maioria era do sexo masculino $(55,7 \%)$, com média de idade \pm desvio-padrão (DP) de 49,72 $\pm 16,18$ anos, era casada ou mantinha união estável (63,6\%). A renda familiar média \pm DP foi de 3,27 \pm 2,90 salários-mínimos e a escolaridade média \pm DP foi de 6,48 \pm 4,29 anos. A tabela 1 detalha as características sociodemográficas da amostra.

As doenças digestivas $(19,6 \%)$ e as doenças respiratórias $(14,4 \%)$ foram as principais responsáveis pelas internações dos pacientes que compuseram a amostra deste estudo (Tabela 2).

A porcentagem de pacientes que relatou falta de apoio social (com menos de quatro parentes e amigos íntimos) foi de 33,8\% ( $N=383$ ). A média do número de parentes e amigos confidentes \pm DP foi de 12,08 $\pm 16,8$. Dos pacientes incluídos na pesquisa, 25,3\% foram considerados como tendo depressão. A tabela 3 descreve a comparação entre deprimidos e não deprimidos em relação às variáveis sociodemográficas e clínicas.

Na Regressão Logística foram incluídas as variáveis: escolaridade, renda familiar, dor, apoio social e gravidade física percebida, que mostraram diferenças estatisticamente significativas entre deprimidos e não deprimidos, mostradas na tabela 3. A comorbidade física objetiva (Índice Charlson de Comorbidade Física) foi incluída nessa análise, mesmo não tendo sido significativa na análise univariada por causa da sua importância do ponto de vista clínico nesse grupo. Na análise multivariada observou-se que a baixa escolaridade [odds ratio (OR): 0,96; intervalo de confiança (IC): 0,89-0,96; $p<0,001$ ], a baixa renda familiar (OR: 0,92; IC: 0,86-0,99; $p=$ 0,018), a maior intensidade de dor (OR: 1,04; IC: 1,00-1,08; $\mathrm{P}$ =0,036), a percepção de falta de apoio social (OR: 2,02; IC: 1,49-2,72; $p<0,001$ ) e a percepção de maior gravidade física (OR: 1,07; IC: 1,02-1,13; $p=0,008$ ) se associaram independentemente à depressão.

Tabela 1. Características sociodemográficas da amostra $(N=1.147)$

\begin{tabular}{|c|c|c|}
\hline Características & $\mathrm{N}(1.147)^{*}$ & $\%$ \\
\hline \multicolumn{3}{|l|}{ Sexo } \\
\hline Masculino & 639 & 55,7 \\
\hline Feminino & 508 & 44,3 \\
\hline \multicolumn{3}{|l|}{ Idade (anos) } \\
\hline 18 a 64 & 920 & 80,2 \\
\hline Maior ou igual a 65 & 227 & 19,8 \\
\hline \multicolumn{3}{|l|}{ Estado civil } \\
\hline Casado(a)/união estável & 729 & 63,6 \\
\hline Solteiro(a) & 168 & 14,6 \\
\hline Separado(a) & 147 & 12,8 \\
\hline Viúvo(a) & 103 & 9 \\
\hline \multicolumn{3}{|c|}{ Renda familiar salários-mínimos* } \\
\hline Menor que 2 & 216 & 22,4 \\
\hline $2 \mathrm{a} 4$ & 537 & 55,7 \\
\hline $5 a 6$ & 109 & 11,3 \\
\hline Maior que 6 & 102 & 10,6 \\
\hline \multicolumn{3}{|l|}{ Escolaridade (anos)* } \\
\hline 0 & 80 & 7 \\
\hline $1 \mathrm{a} 4$ & 415 & 36,2 \\
\hline 5 a 8 & 313 & 27,3 \\
\hline 8 ou mais anos & 326 & 28,4 \\
\hline
\end{tabular}

*Os valores que, somados, não atingirem o $\mathrm{N}$ total da amostra são decorrentes do não preenchimento completo pelo entrevistado, por desconhecimento ou recusa.

Tabela 2. Doenças que motivaram a internação, de acordo com a CID-10 (Classificação Internacional de Doenças, 10a revisão)

\begin{tabular}{lcc}
\hline Motivo da internação & $\mathrm{N}(1.147)$ & $\%$ \\
\hline Digestivas & 225 & 19,6 \\
Respiratórias & 165 & 14,4 \\
Cardíacas/circulatórias & 157 & 13,7 \\
Hematológicas/imunitárias & 146 & 12,7 \\
Endócrinas/nutricionais/metabólicas & 101 & 8,8 \\
Neurológicas & 87 & 7,6 \\
Neoplasias & 60 & 5,2 \\
Osteomusculares/tecido conjuntivo & 43 & 3,7 \\
Mal-definidas & 42 & 3,7 \\
Geniturinárias & 37 & 3,2 \\
Infecciosas/parasitárias & 35 & 3,1 \\
Pele/subcutâneo & 11 & 1 \\
Causas externas & 9 & 0,8 \\
Lesões/envenenamentos & 8 & 0,7 \\
\hline
\end{tabular}


Tabela 3. Análise univariada comparando deprimidos e não deprimidos internados em enfermarias de clínica médica em relação às variáveis sociodemográficas, clínicas e fatores psicossociais $(\mathrm{N}=1.147)$

\begin{tabular}{|c|c|c|c|}
\hline \multirow{2}{*}{ Variável* } & \multicolumn{2}{|c|}{ Depressão? ${ }^{\dagger}$} & \multirow{2}{*}{$P$} \\
\hline & Não & Sim & \\
\hline \multicolumn{4}{|l|}{ Sexo (\%) } \\
\hline Homens & 76,1 & 23,9 & \multirow{2}{*}{0,17} \\
\hline Mulheres & 72,4 & 27,6 & \\
\hline Idade & $49,84 \pm 16,29$ & $49,22 \pm 15,53$ & 0,56 \\
\hline Escolaridade (anos) & $6,83 \pm 4,46$ & $5,53 \pm 3,66$ & $<0,001$ \\
\hline Renda familiar ${ }^{\ddagger}$ & $3,49 \pm 2,95$ & $2,69 \pm 2,74$ & $<0,001$ \\
\hline Rede de apoio social $\left.\right|^{5}(\%)$ & 70,9 & 54,1 & $<0,001$ \\
\hline \multicolumn{4}{|l|}{ Variáveis clínicas } \\
\hline ॥ Gravidade física objetiva & $1,13 \pm 1,46$ & $1,01 \pm 1,4$ & 0,23 \\
\hline 'Gravidade física percebida & $6 \pm 2,95$ & $6,83 \pm 3,02$ & $<0,001$ \\
\hline Dor ** & $4,12 \pm 3,79$ & $5,20 \pm 3,92$ & $<0,001$ \\
\hline
\end{tabular}

* Os dados estão expressos em média \pm desvio-padrão, exceto quando especificados de outra forma.

† Foram considerados como tendo depressão aqueles pacientes que pontuaram acima de 10 na Subescala Cognitivo-afetiva (primeiros 13 itens - BDI-13) do Inventário Beck de Depressão. ‡ Renda familiar em salários-mínimos.

§ Responderam um número maior que 3 à pergunta: "Com quantos parentes e amigos você se sente à vontade e pode falar sobre tudo ou quase tudo?".

$\|$ Índice Charlson de Comorbidade Física.

"Respondendo: "De zero a dez, qual número melhor descreve a gravidade de sua doença no momento? Zero seria sem doença e dez seria a doença mais grave possível".

** Respondendo: "De zero a dez qual número melhor descreve sua dor agora? Zero seria sem dor e dez seria a dor mais forte possível".

\section{DISCUSSÃO}

Este é um dos primeiros estudos realizados em nossa comunidade que avalia tanto a comorbidade psiquiátrica como fatores psicossociais, dor e outros aspectos clínicos em pacientes internados por doenças físicas. Cerca de um quarto dos pacientes internados por doenças físicas nas Enfermarias de Clínica Médica do HU-UFSC que compuseram a nossa amostra tinham depressão (25,3\%). Verificando-se a correlação entre as variáveis, observou-se que os deprimidos tinham menor escolaridade e menor renda familiar, menor apoio social, maior gravidade física percebida, assim como maiores níveis de dor. Não houve diferença estatisticamente significativa em relação a sexo, idade e comorbidade física objetiva. Na análise multivariada, menor escolaridade, menor renda familiar, falta de apoio social, maior intensidade da dor e percepção de maior gravidade física associaram-se significativamente à depressão, mesmo após ajustar para fatores de confusão. Esses achados estão condizentes com outros estudos 2,16,28,29.

Diferentemente de nossos achados, lliffe et al. ${ }^{15}$ verificaram que a intensidade da dor não estava estatisticamente relacionada com a depressão, mesmo encontrando prevalência semelhante de depressão na amostra analisada (19\%). Esse mesmo estudo revelou uma associação entre depressão e risco de isolamento social. Talvez a diferença encontrada seja devida à diferença de amostras, sendo a amostra de lliffe exclusivamente de pessoas acima de 65 anos, não hospitalizadas e independentes para autocuidado ${ }^{15}$.

Existem limitações para este estudo, que são as seguintes: 1) O diagnóstico da depressão foi feito por meio de uma escala, e não por um psiquiatra. Contudo, os estudos que associam depressão com maior mortalidade utilizaram essa mesma escala, o BDI ${ }^{11,12}$; 2) Nossa amostra foi composta por pacientes internados em um hospital universitário, com nível de atenção terciário. É possível que pacientes na atenção primária ou secundária tenham padrões diferentes; 3 ) Por ser um estudo transversal, não é possível estabelecer relação de causa e efeito; 4) Pacientes muito graves e incapacitados (por exemplo, com dispneia intensa) foram excluídos por não conseguirem responder à entrevista, portanto não estão representados na nossa amostra. Seria importante que estudos futuros desenvolvessem métodos para detectar depressão, dor e apoio social nesse grupo; 5) O apoio social foi avaliado por meio de pergunta direta, o que pode gerar questionamentos quanto à sua validade. Cabe ressaltar que essa pergunta na realidade é um instrumento já validado, inclusive em uma amostra brasileira ${ }^{25}$. Apesar de existirem questionários mais completos para essa avaliação, a simplicidade e a rapidez dos instrumentos são fundamentais em pesquisas realizadas nas enfermarias de clínica médica, uma vez que os pacientes estão muito debilitados e necessitam de tempo para fazer exames e o tratamento da doença de base.

Como significado clínico para esses achados, observa-se a importância de questionar sobre rede de apoio social e sintomas sugestivos de depressão em indivíduos com doenças físicas, sobretudo naqueles com maiores níveis de dor, pois esses fatores são frequentes e podem estar influindo em seu quadro clínico. Simples perguntas, como "Com quantos parentes e amigos você se sente à vontade e pode falar sobre tudo ou quase tudo?" Ou "De zero a dez, qual o número que descreve sua dor agora?", podem ajudar a detectar fatores relacionados e a suspeitar da presença de depressão, per- 
mitindo, assim, o diagnóstico e o tratamento precoce dessa doença.

Uma pesquisa revelou que em idosos o tratamento da depressão e orientações para lidar com a doença física e aprimorar a capacidade de realizar atividades diárias levaram à percepção de maior apoio social, melhoria das condições gerais do paciente e sua qualidade de vida relacionada à saú$\mathrm{de}^{30}$. Além disso, vem sendo demonstrado que o tratamento da depressão pode, além de reduzir os sintomas depressivos, levar a uma redução de marcadores inflamatórios.? É possível que, por meio do estudo das vias de ativação de imunidade mediada por células e outros mecanismos que estimulam a manutenção de processos inflamatórios possam ser gerados dados que ajudem a elucidar a alta associação entre dor, depressão e diversas doenças sistêmicas ${ }^{5}$. Contudo, apesar das novas vias que vêm sendo propostas, não se pode esquecer da importância de um bom manejo clínico desses pacientes, que envolve aspectos do correto tratamento da dor, da relação médico-paciente e da rede de apoio social.

\section{CONCLUSÃO}

Os pacientes internados nas Enfermarias de Clínica Médica do HU-UFSC que apresentavam depressão (BDI-13 > 10) relataram maiores níveis de dor e mais falta de apoio social, mesmo após controlar para variáveis confundidoras sociodemográficas e clínicas. Estudos longitudinais podem ajudar a elucidar a natureza dessa associação.

\section{CONTRIBUIÇÕES INDIVIDUAIS}

Camila Andrade Marques - Coleta, análise dos dados e redação do manuscrito.

Betina Stefanello e Camila Nemoto de Mendonça - Coleta, análise dos dados e revisão do manuscrito.

Letícia Maria Furlanetto - Elaboração do projeto, supervisão da coleta, análise dos dados e redação do manuscrito.

\section{CONFLITOS DE INTERESSE E SUPORTE FINANCEIRO}

Nenhum autor tem conflitos de interesse.

Este trabalho teve apoio financeiro do CNPq, por meio de Bolsa de Iniciação Científica (PIBIC) concedida às três primeiras autoras (Camila Andrade Marques, Betina Stefanello, Camila Nemoto de Mendonça).

\section{AGRADECIMENTOS}

Agradecemos aos outros integrantes do grupo de pesquisa do LETH (Laboratório de Estudos em Transtornos de Humor) que participaram da coleta de dados.

\section{REFERÊNCIAS}

1. Williams LJ, Pasco JA, Jacka FN, Dodd S, Berk M. Pain and the relationship with mood and anxiety disorders and psychological symptoms. J Psychosom Res. 2012;72(6):452-6.

2. Castro M, Kraychete D, Daltro C, Lopes J, Menezes R, Oliveira I. Comorbid anxiety and depression disorders in patients with chronic pain. Arq Neuropsiquiatr. 2009;67(4):982-5.

3. Katon W, Unutzer J, Russo J. Major depression: the importance of clinical characteristics and treatment response to prognosis. Depress Anxiety. 2010;27(1):19-26.

4. Van't Land H, Verdurmen J, Ten Have M, Van Dorsselaer S, Beekman A, De Graaf R. The association between arthritis and psychiatric disorders; results from a longitudinal population-based study. J Psychosom Res. 2010;68(2):187-93.

5. Leonard B, Maes M. Mechanistic explanations how cell-mediated immune activation, inflammation and oxidative and nitrosative stress pathways and their sequels and concomitants play a role in the pathophysiology of unipolar depression. Neurosci Biobehav Rev. 2012;36(2):764-85.

6. Kojima M, Kojima T, Suzuki S, Oguchi T, Oba M, Tsuchiya H, et al. Depression, inflammation, and pain in patients with rheumatoid arthritis. Arthritis Rheum. 2009;61(8):1018-24.

7. Thornton LM, Andersen BL, Schuler TA, Carson WE 3rd. A psychological intervention reduces inflammatory markers by alleviating depressive symptoms: secondary analysis of a randomized controlled trial. Psychosom Med. 2009;71(7):715-24.

8. Beesdo K, Jacobi F, Hoyer J, Low NC, Hofler M, Wittchen HU. Pain associated with specific anxiety and depressive disorders in a nationally representative population sample. Soc Psychiatry Psychiatr Epidemiol. 2010;45(1):89-104.

9. Richardson EJ, Ness TJ, Doleys DM, Banos JH, Cianfrini L, Richards JS. Depressive symptoms and pain evaluations among persons with chronic pain: catastrophizing, but not pain acceptance, shows significant effects. Pain. 2009;147(1-3):147-52.

10. Citero Vde A, Levenson JL, MCClish DK, Bovbjerg VE, Cole PL, Dahman BA, et al. The role of catastrophizing in sickle cell disease: the PiSCES project. Pain. 2007;133(1-3):39-46.

11. Carney RM, Freedland KE. Treatment-resistant depression and mortality after acute coronary syndrome. Am J Psychiatry. 2009;166(4):410-7.

12. Frasure-Smith N, Lesperance F, Gravel G, Masson A, Juneau M, Talajic M, et al. Social support, depression, and mortality during the first year after myocardial infarction. Circulation. 2000;101(16):1919-24.

13. Trivedi RB, Blumenthal JA, O'Connor C, Adams K, Hinderliter A, Dupree C, et al. Coping styles in heart failure patients with depressive symptoms. J Psychosom Res. 2009;67(4):339-46.

14. Pearson CR, Micek MA, Pfeiffer J, Montoya P, Matediane E, Jonasse T, et al. One year after ART initiation: psychosocial factors associated with stigma among HIV-positive Mozambicans. AIDS Behav. 2009;13(6):1189-96.

15. Iliffe S, Kharicha K, Carmaciu C, Harari D, Swift C, Gillman G, et al. The relationship between pain intensity and severity and depression in older people: exploratory study. BMC Fam Pract. 2009;10:54.

16. Jenks Kettmann JD, Altmaier EM. Social support and depression among bone marrow transplant patients. J Health Psychol. 2008;13(1):39-46.

17. McKenzie M, Clarke DM, McKenzie DP, Smith GC. Which factors predict the persistence of DSM-IV depression, anxiety, and somatoform disorders in the medically ill three months post hospital discharge? J Psychosom Res. 2009;68(1):21-8. 
18. Lyness JM, Yu Q, Tang W, Tu X, Conwell Y. Risks for depression onset in primary care elderly patients: potential targets for preventive interventions. Am J Psychiatry. 2009;166(12):1375-83.

19. Folstein MF, Folstein SE. "Mini-Mental State". A practical method for grading the cognitive state of patients for the clinician. J Psychiatr Res. 1975;12:189-98.

20. O'Connor DW, Pollitt PA, Hyde JB, Fellows JL, Miller ND, Brook CP, et al. The reliability and validity of the Mini-Mental State in a British community survey. J Psychiatr Res. 1989;23(1):87-96.

21. Bertolucci PH, Brucki SM, Campacci SR, Juliano Y. [The Mini-Mental State Examination in a general population: impact of educational status]. Arq Neuropsiquiatr. 1994;52(1):1-7.

22. Beck AT, Ward CH, Mendelson M, Mock J, Erbaugh J. An inventory for measuring depression. Arch Gen Psychiatry. 1961;:4:561-71.

23. Furlanetto LM, Mendlowicz MV, Romildo Bueno J. The validity of the Beck Depression Inventory-Short Form as a screening and diagnostic instrument for moderate and severe depression in medical inpatients. J Affect Disord. 2005;86(1):87-91.

24. Jensen MP, Karoly P, Braver S. The measurement of clinical pain intensity: a comparison of six methods. Pain. 1986;184:117-26.
25. Chor D, Griep RH, Lopes CS, Faerstein E. [Social network and social support measures from the Pro-Saude Study: pre-tests and pilot study]. Cad Saude Publica. 2001;17(4):887-96.

26. Brugha TS, Weich S, Singleton N, Lewis G, Bebbington PE, Jenkins R, et al. Primary group size, social support, gender and future mental health status in a prospective study of people living in private households throughout Great Britain. Psychol Med. 2005;35(5):705-14.

27. Charlson ME, Pompei P, Ales KL, MacKenzie CR. A new method of classifying prognostic comorbidity in longitudinal studies: development and validation. J Chron Dis. 1987;40(5):373-83.

28. Stalnacke BM. Relationship between symptoms and psychological factors five years after whiplash injury. J Rehabil Med. 2009;41(5):353-9.

29. Marlow RA, Kegowicz CL, Starkey KN. Prevalence of depression symptoms in outpatients with a complaint of headache. J Am Board Fam Med. 2009;22(6):633-7.

30. Chan SW, Chiu HF, Chien WT, Goggins W, Thompson D, Hong B. Predictors of change in health-related quality of life among older people with depression: a longitudinal study. Int Psychogeriatr. 2009;21(6):1171-9. 